Original paper

\title{
Synchrotron X-ray microbeams: A promising tool for drug-resistant epilepsy treatment
}

\author{
F. Studer a, b, R. Serduc a, b, B. Pouyatos ${ }^{\text {d }}$, T. Chabrol a, b, E. Bräuer-Krisch ${ }^{\text {e }}$, M. Donzelli ${ }^{\text {e }}$, \\ C. Nemoz ${ }^{\text {e }}$, J.A. Laissue ${ }^{f}$, F. Estève ${ }^{\text {a, b, c }}$, A. Depaulis ${ }^{\text {a, b, c, * }}$ \\ anserm, U836, F-38000 Grenoble, France \\ ${ }^{\mathrm{b}}$ Univ. Grenoble Alpes, Grenoble Institut des Neurosciences, F-38000 Grenoble, France \\ ${ }^{c} \mathrm{CHU}$ de Grenoble, F-38000 Grenoble, France \\ d SynapCell SAS, 38700 La Tronche, France \\ e European Synchrotron Research Facility, Biomedical Beamline ID17, Grenoble, France \\ ${ }^{\mathrm{f}}$ University of Bern, Switzerland
}

\section{A R T I C L E I N F O}

\section{Article history:}

Received 8 January 2015

Received in revised form 7 April 2015

Accepted 9 April 2015

Available online 29 April 2015

\section{Keywords:}

Epilepsy

Radiosurgery

Synchrotron X-ray microbeams

Animal models

\begin{abstract}
A B S T R A C T
Epilepsy is one of the most important neurological diseases. It concerns about $1 \%$ of the population worldwide. Despite the discovery of new molecules, one third of epileptic patients are resistant to antiepileptic drugs and among them only a few can benefit from resective surgery. In this context, radiotherapy is an interesting alternative to the other treatments and several clinical devices exist (e.g., Gamma Knife ${ }^{\circledR}$ ). The European Synchrotron Radiation Facility offers the possibility to develop new methods of radiosurgery and to study their antiepileptic effects. Here, we discuss several studies that we performed recently to test and try to understand the antiepileptic effects of X-ray synchrotron microbeams in different animal models of epilepsy. We showed a decrease of seizures after Interlaced Microbeam Radiotherapy (IntMRT) of the somatosensory cortex, known as the seizure generator, in a genetic model of absence epilepsy. These antiepileptic effects were stable over 4 months and with low tissular and functional side-effects. The irradiated pyramidal neurons still displayed their physiological activity but did not synchronize anymore. We also obtained a lasting suppression of seizures after IntMRT of the dorsal hippocampus in a mouse model of mesiotemporal lobe epilepsy. However, an important variability of antiepileptic efficiency was observed probably due to the small size of the targeted structure. Despite these encouraging proofs-of-concepts, there is now a need to adapt IntMRT to other models of epilepsy in rodents which are close to refractory forms of epilepsy in human patients and to implement this approach to non-human primates, before moving to clinical trials.

() 2015 Published by Elsevier Ltd on behalf of Associazione Italiana di Fisica Medica. This is an open access article under the CC BY-NC-ND license (http://creativecommons.org/licenses/by-nc-nd/4.0/).
\end{abstract}

\section{The epilepsies and the need for new therapies}

Epilepsy is a devastating multi-causal chronic disease characterized by recurrent spontaneous seizures and affects about $1 \%$ of the population worldwide. This represents about 3 million people in Europe which implies an annual cost of 15.5 billion euros [1]. Although epilepsy is defined by the presence of recurrent seizures, it is often associated with comorbid abnormalities in cognition,

\footnotetext{
* Corresponding author. Grenoble - Institut des Neurosciences, Centre de recherche Inserm U 836, Université Joseph Fourier - Faculté de Médecine, Domaine de la Merci, Chemin Fortuné Ferrini, 38700 La Tronche, France. Tel.: +33045652 06 65; fax: +330456520669.

E-mail address: antoine.depaulis@ujf-grenoble.fr (A. Depaulis).
}

psychiatric status, and social-adaptive behavior, as well as with an increased risk of mortality. This is especially true in drug-resistant epilepsies, which represent a socioeconomic burden at the individual, familial, societal and political levels in all countries across the globe. Drug-resistant epilepsies account for about one third of adult epileptic patients. Among them, some may benefit from resective surgery of the epileptogenic zone. Indeed, complete seizure freedom can be obtained by neurosurgical resective surgery with a rate of success of about $42 \% 5$ years after resection to $33 \% 10$ years later [2]. Resective surgery is currently the gold-standard treatment capable of suppressing drug-resistant seizures [3]. But surgery requires that the epileptic zone is (i) focal, (ii) clearly identified and (iii) can be safely removed [4]. This is a rather rare combination and only a limited number of patients can indeed benefit from such a surgical approach. The development of 
alternative surgical approaches for challenging brain areas could increase the number of drug-resistant patients that could be cured from their focal epilepsy. In this context, our consortium has investigated for the last 5 years the effects of synchrotron microbeam-based therapies to suppress seizures in animal models. In this chapter, we review our work and discuss the perspectives of this approach.

\section{The use of microbeams}

Radiosurgery represents one of the most promising alternative to resective surgery. Indeed, modern radiosurgical devices such as Gamma Knife $^{\circledR}$ and Cyberknife ${ }^{\circledR}[5]$ have provided satisfying results in treating various types of epilepsies associated with arteriovenous malformations, cavernomas, tumors, mesio-temporal sclerosis and hypothalamic hamartomas (see Ref. [6] for review). However, the reported failures in radiosurgical treatment of epileptic foci are often correlated with the radiosensitivity of normal tissues surrounding the lesion, which sets the limit to the radiation dose that can be safely delivered within the target $[7,8]$. The lateral dose gradients from the irradiated target to surrounding sensitive structures which are achievable with high-energy photons (MeV range) display a broad distribution of dose (Fig. 1), thus preventing delivery of high enough and curative doses to the target without injuring functional adjacent structures. The low-energy photons (keV) generated by 3rd generation synchrotron sources offer an adequate dose rate, energy spectrum and a minimal beam divergence which allows the deposition of extremely steep lateral dose fall-off (Fig. 1) [9]. Therefore, synchrotron X-ray radiosurgery provides sufficient precision to perform experimental studies in animal models of epilepsy, which is unachievable using conventional megavoltage radio-oncologic devices used in hospitals.

The use of spatial fractionation of ionizing radiation in the microscopic range was first reported at the end of the fifties [10-13]. A $25 \mu \mathrm{m}$-wide deuteron microbeam failed to elicit visible cerebral damage in mice unless absorbed doses were over $3000 \mathrm{~Gy}$; the deuterons, however, reached only $1.5 \mathrm{~mm}$ tissue depth. The recent availability of microplanar 50-150 keV synchrotrongenerated $\mathrm{x}$-ray beams (microbeams or $\mathrm{MB}$ ) produces sharply defined beam edges deep in the tissues and with a high dose rate [14]. This has led to investigate the effects of MB on murine brains, using arrays of quasi parallel 25-75 $\mu \mathrm{m}$-wide beam slices spaced $50-200 \mu \mathrm{m}$ on center [15]. The tissue lesions resembled those induced by deuteron MB [11,12]. Surprisingly, animals withstood absorbed doses of hundreds, even thousands of gray from x-rays delivered to microscopic slices of their brains [16]. Tissue damage (i.e., cellular death) was confined to the MB paths. For example, adult cerebellum of pigs irradiated as weanling piglets showed stripes of cell loss restricted to the MB tracts [17] (Fig. 2). All irradiated pigs developed normally and never displayed any behavioral or developmental adverse effects.

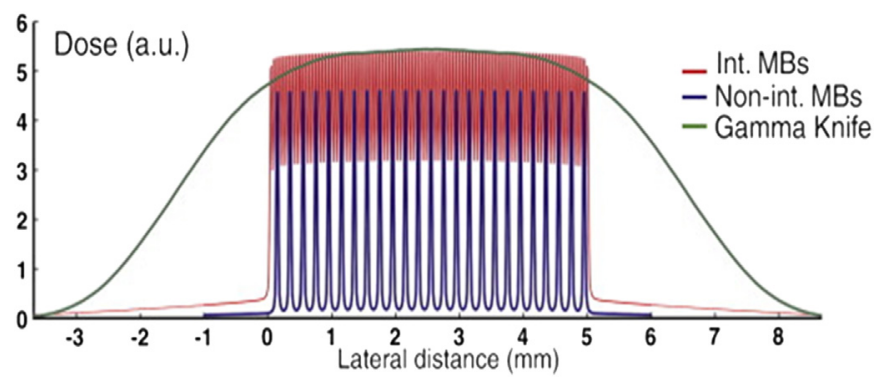

Figure 1. Lateral dose-profiles for a $5 \mathrm{~mm}$-wide irradiation field produced by interlaced and non-interlaced MB and by a Leksell Perfexion ${ }^{\circledR}$ Gamma-Knife.

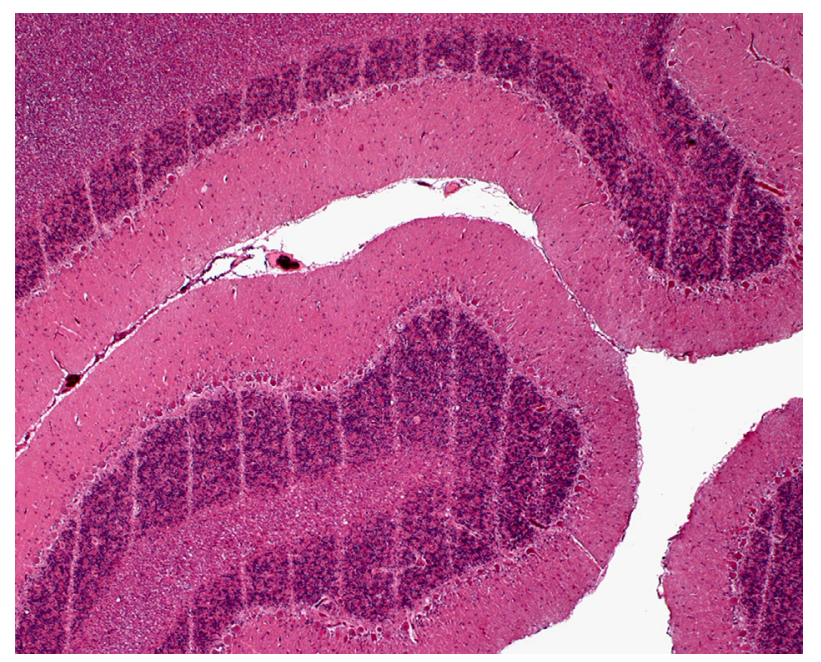

Figure 2. Histological section of pig cerebellum 15 months after MB (20-30 $\mu$ m wide $210 \mu \mathrm{m}$ apart) irradiation performed with an entrance dose of $300 \mathrm{~Gy}$ (HE staining).

Recently, we introduced a novel use of MB: by rotating the brain around a center of rotation using a goniometer, we precisely interlaced $\mathrm{MB}$ to deposit a high homogeneous radiation dose into discrete brain regions [18], with no extensions on neighboring tissue [18] (Fig. 3). This irradiation procedure, which allows a lateral dose fall-off about 200 times greater than conventional radiotherapy [18], was called Interlaced Microbeam Radiotherapy (IntMRT). The submillimetric precision of targeting, combined with the preservation of surrounding tissues, makes IntMRT clinically attractive for all pathologies that require circumscribed destruction, inactivation and/or disconnection of small brain regions, even if close to eloquent or vital structures.

\section{First proof of concept of IntMRT in a genetic model of epilepsy in the rat}

\section{Short term functional effects}

The efficiency of IntMRT is currently verified in rodent brain tumor treatment but this novel approach of high dose delivery appeared relevant for non-cancerous brain disease therapies. In 2010, we established a collaboration which aimed at evaluating IntMRT efficacy on epileptic tissue. Our working hypothesis was based on the fact that several studies in patients $[19,20]$ and animal models [21-23] suggested that an antiepileptic effect could be obtained even if the irradiation did not result in necrosis of the targeted structure. This non-necrotizing radiotherapeutic effect was believed to be caused by biochemical alterations and neuromodulation, although the cellular mechanisms remained elusive [20,24]. One of the prerequisites was to target an epileptic tissue with well-identified neurons generating seizures and presenting no cell loss and/or sclerosis to avoid confounding factors, thus allowing the assessment of radiation-induced changes. For these reasons, we chose to first perform IntMRT in the Genetic Absence Epilepsy Rats from Strasbourg (GAERS) [25]. The GAERS has emerged as a well-recognized and studied rat model for idiopathic generalized epilepsy with absence seizures. Many studies have characterized its behavioral, electrophysiological, pathophysiological and pharmacological features, which closely resemble human absence seizures $[26,27]$. GAERS display very stable spontaneous spike-and-wave discharges (SWD) on the cortical electroencephalogram (EEG) accompanied by simultaneous behavioral arrest, characteristic of absence seizures. In addition, the model allows both long term 
A

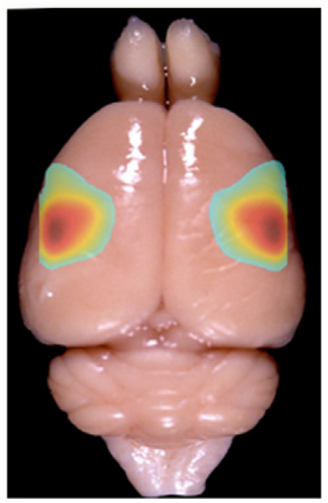

B

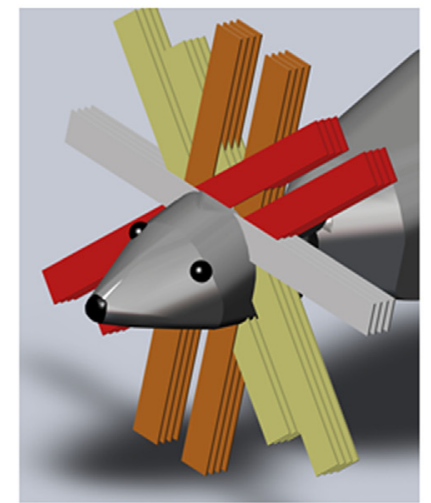

C

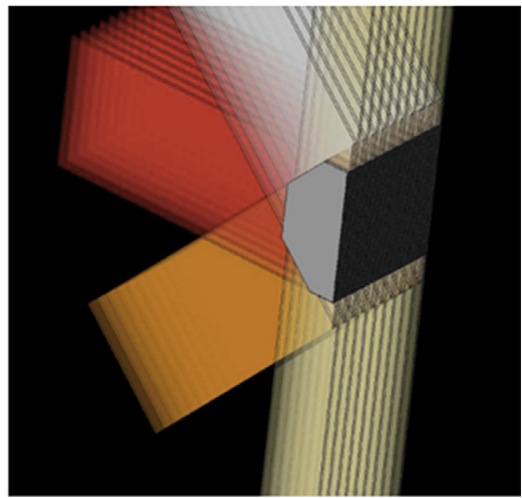

D

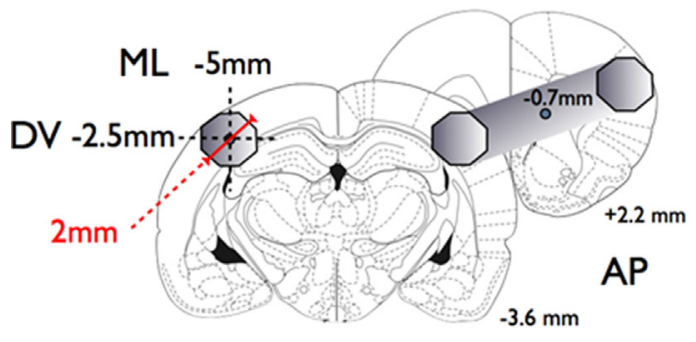

E

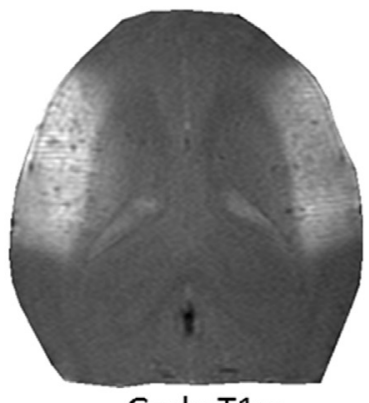

Gado T1w

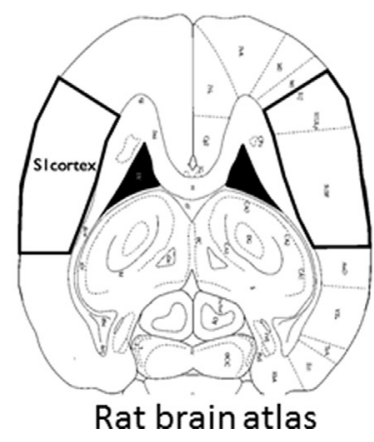

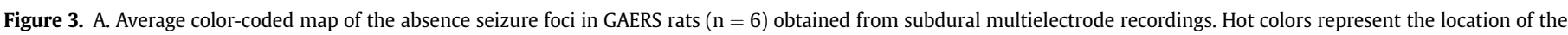

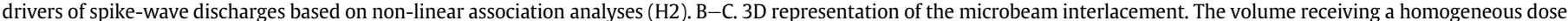

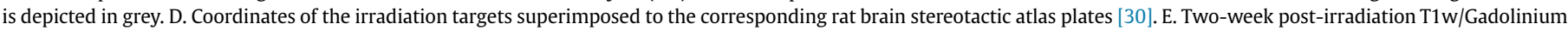

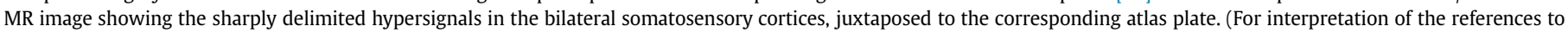
colour in this figure legend, the reader is referred to the web version of this article.)

seizure monitoring and access to the ictal activity of individual neurons [28].

Recently, we showed in the GAERS that the SWD are initiated in the somato-sensory cortex and then spread rapidly to the motor cortex and the ventrobasal thalamus $[28,29]$. We therefore targeted these three brain regions. In order to precisely irradiate the brain volume of each of these regions, we positioned anaesthetized GAERS rats on a Plexiglas stereotactic frame mounted on micrometric motors, allowing movements and rotations in the 3 dimensions. The bregma, at the junction of the frontal and parietal bones, was clearly visualized on the x-ray reconstructed image of the rat's head and used to define the coordinates [30] of irradiation fields. Then, the beam was spatially fractionated into an array of parallel microbeams ( $50 \mu \mathrm{m}$ wide, $200 \mu \mathrm{m}$ on-center distance) by a multislit collimator. Each side of the targeted brain regions was irradiated using 4 ports separated by a $45^{\circ}$ angle and a $50 \mu \mathrm{m}$ step, to generate a solid dose deposition at the interlaced region (Fig. 3). The nominal dose deposited within the target was fixed at $200 \mathrm{~Gy}$ (according to Monte Carlo simulations [31]). In each animal, we verified the correct targeting by $\mathrm{T}_{1}$-weighed magnetic resonance imaging (MRI) within the two weeks that followed IntMRT and examined their general behavior by standard tests to detect possible side effects. We also euthanized some irradiated animals for histological analysis, using Nissl stains and stains for myelin sheaths. Outside the interlaced target, the microbeam tracks were visible in the striatum and the S1 cortex (Fig. 4B) on histological brain sections obtained two months after irradiation, as thin clear stripes alternating with darker, nominally non-irradiated tissue swathes $\approx 200 \mu \mathrm{m}$ spaced apart. Radiation effects were also confirmed on immuno-labeled slices for NeuN, GFAP, Type IV collagen, RECA and GLUT-1, respectively markers of neurons, astrocytes, basal lamina, angiogenesis and glucose transporter. MRI controls done 2 weeks after irradiation confirmed the accuracy of radiation targeting and the minimal changes in surrounding tissues, as shown by localized increase in contrast-agent diffusion on MRI (Figs. 3E and 4A). Similarly, histopathological controls revealed minor structural tissue disruptions in the short-term post-irradiation phase (2 months) (Fig. 4B). It is important to note that although eloquent cortex was targeted bilaterally, no significant behavioral alterations were measured (Rotarod ${ }^{\circledR}$, open-field) in the acute (2 weeks) post-irradiation phase. Altogether these controls confirmed that IntMRT has limited short-term deleterious effects on brain tissue and does not lead to strong motor or cognitive impairments up to two months after irradiation.

During the 4 months that followed irradiation, we monitored absence seizures weekly, in freely-moving animals, by recording local-field potentials (LFP) from depth electrodes implanted in the three target regions. These recordings showed that MB irradiation suppressed SWD when the somatosensory cortex was irradiated (Fig. 3C). While non-irradiated cortical and thalamic regions could still produce epileptic discharges, the irradiated volume became isolated from the pathological activity. In contrast, MB irradiation of the motor cortex or ventrobasal thalamus did not significantly suppress SWD [25]. The mechanisms of the functional exclusion of the somatosensory cortex were further investigated by individually recording irradiated neurons two months after irradiation using in vivo intracellular electrophysiological recordings, as previously used [28]. This approach revealed that the irradiated pyramidal neurons were strongly hyperpolarized, displayed a decreased excitability and a reduction of spontaneous synaptic activities [25] (Fig. 4C). These functional alterations may explain the suppression of paroxysmal oscillations within irradiated cortical networks 
A

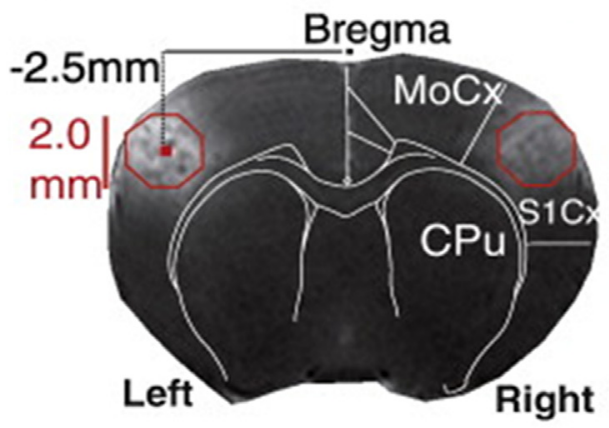

B

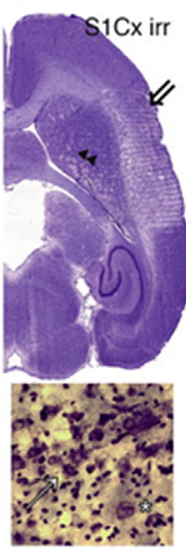

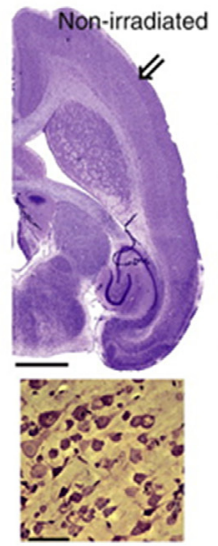

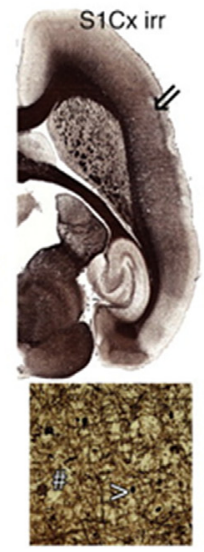

C

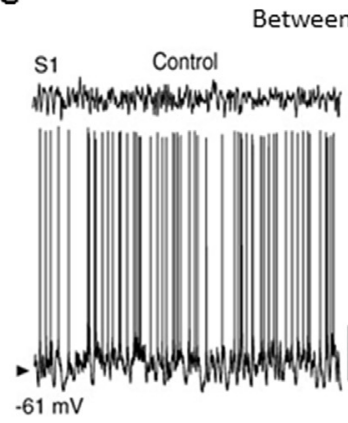

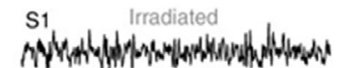

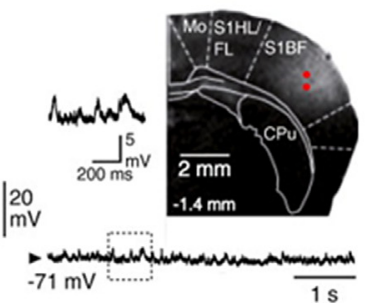

D

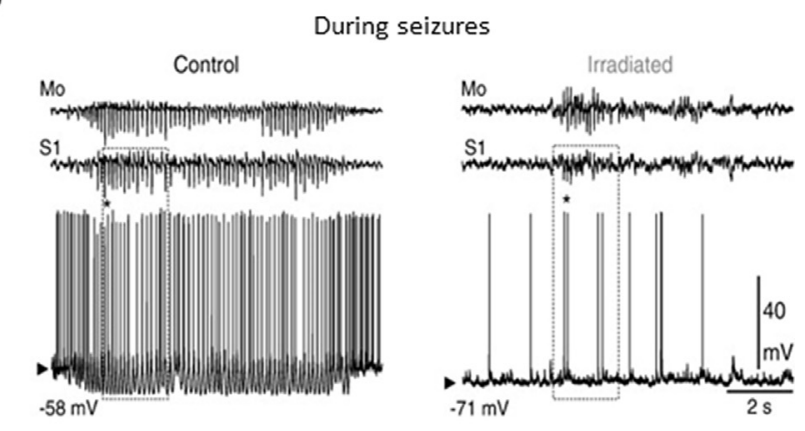

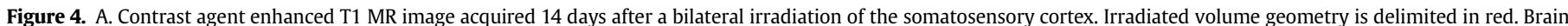

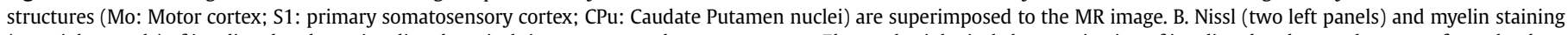

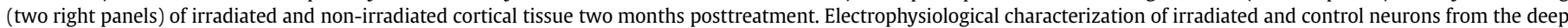

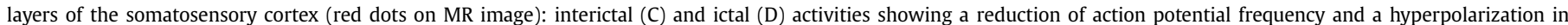

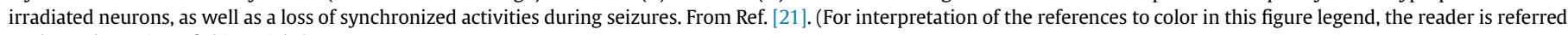
to the web version of this article.)

(Fig. 4D). This pioneering work provides the first post-irradiation electrophysiological recordings of individual neurons. As such, these recordings are a preliminary and critical step towards understanding how ionizing radiation energy delivery impacts neuronal physiology and physiopathology. Indeed, our data should provide clues on how radiation dose should be delivered for targeting epileptic neuronal networks.

\section{Long-term histological effects}

Our experiment provided evidence of moderate histological consequences at 2 months post-irradiation. Nevertheless, longterm risks (one year or more) associated with the delivery of quasi-seamless doses into restricted brain regions should not be minimized. Indeed, the extent of normal tissue damage induced by moderate doses delivered by MB depends to a large extent on spatial fractionation. IntMRT delivers a high radiation dose almost homogeneously to a submillimetric well-delimited brain volume [18]. In a normal brain, such lesions display short-term MRI features indicative of a disruption of the blood-brain barrier [32]. Few data are yet available on long-term consequences of IntMRT in the brain. Even low doses of seamless irradiation delivered by a single broad beam elicit late vascular and parenchymal necrosis in the central nervous system of laboratory animals, mostly as a consequence of arterionecrosis [33]. Stereotactic irradiation of the frontal cerebral lobes of normal rats with 2 or $3 \mathrm{~mm}$ sized collimators and doses of 30-50 Gy result in radiation necrosis about 19 months after exposure [34]. Such observations temper against premature assessments of damage to the central nervous system by homogeneous irradiations. Preliminary data in two rats revealed delayed focal necrotizing arteritic lesions and ensuing cerebral infarction in the target regions 6 months after IntMRT that likely developed weeks before the rats' death (Fig. 5). We can hypothesis that by irradiating a smaller brain volume it should be possible to avoid the biggest brain vessels and prevent these vascular damages.

\section{Application of IntMRT to other animal models}

GAERS models absence-epilepsy, a form of epilepsy that is well controlled by anti-epileptic drugs, disappears in most patients at adulthood and probably would never be a candidate for clinical IntMRT. Therefore, we are currently applying IntMRT to other animal models of epilepsy with focal seizures and that are reminiscent of epilepsies that would be candidates for such therapies.

\section{Mesiotemporal lobe epilepsy model in mice}

We first applied IntMRT to a mouse model of mesiotemporal lobe epilepsy (MTLE). This syndrome classically occurs in human patients several years after an initial precipitating insult (e.g., complex febrile seizures). The first seizures are generally well controlled by antiepileptic drugs (AED) but often become medically refractory. The focal seizures are characterized by a motionless stare and oro-alimentary automatisms (e.g., lip smacking, chewing) 
A

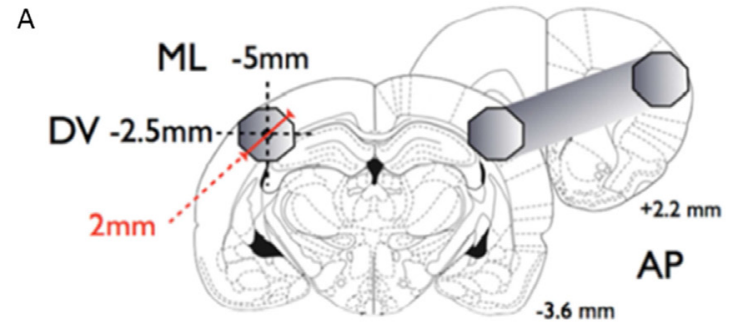

C

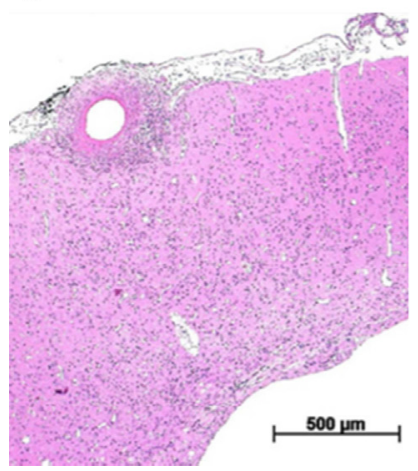

B

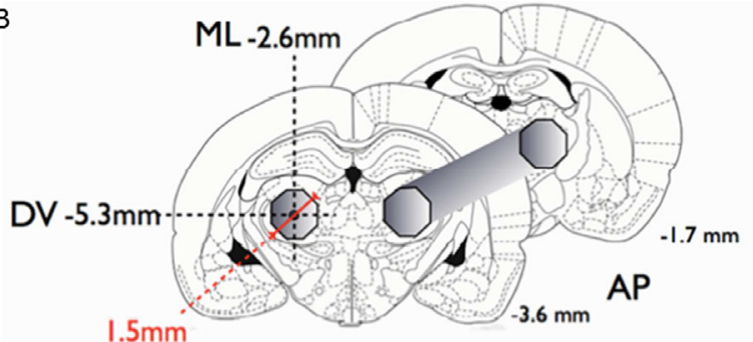

$E$
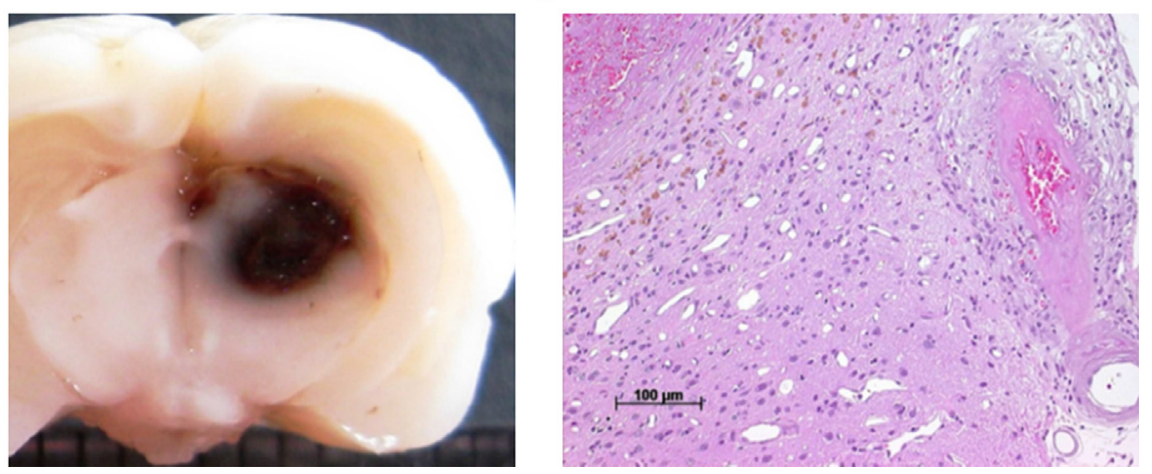

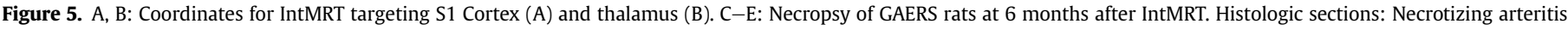

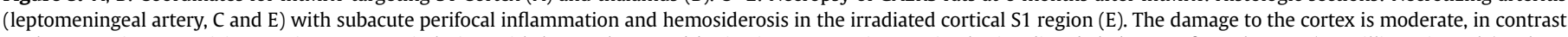

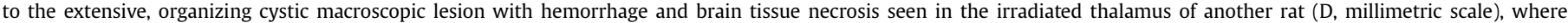
fibrinoid vascular changes were also present.

associated with a progressive clouding of consciousness but rarely generalized. In addition to these clinical features, the MTLE syndrome is generally associated with a hippocampal sclerosis, mainly characterized by a selective neuronal loss in the CA1/CA3 and hilus area and a proliferation of astroglial cells within the Ammon's horn of the hippocampus and the hilus of the dentate gyrus (for review see [35]).

MTLE is the principal indication for resective surgery. Actually, about $2 / 3$ of patients that undergo this procedure are patient suffering drug-refractory MTLE [36]. The procedure consists in surgically removing the seizure onset zone, generally the hippocampus, after functional exploration using depth electrodes. This surgery has an excellent track record showing that the hippocampus can be safely removed with minimal deleterious functional and behavioral consequences. Thus, MTLE appeared as the most logical target for a proof-of-concept application of IntMRT on drugresistant focal epilepsy.

Several animal models of this syndrome have been developed in rats and mice (for review see Depaulis \& Hamelin2015 [37]). Among them intrahippocampal injection of kainate in mice was shown to induce ipsilateral excitotoxic damages (i.e., cell loss in CA1, CA3 and dentate gyrus hilus) during the $2-3$ weeks that follow the injection, as well as proliferation and hypertrophy of astrocytes and microglia, sprouting of the mossy fibers and dispersion and hypertrophy of neurons of the dentate gyrus [38-40]. These changes are restricted to the injected hippocampus and show a great similarity to the most severe type of hippocampal sclerosis, observed in up to $80 \%$ of mesial-temporal lobe resections from MTLE patients [35]. In this model, spontaneous seizures with a hippocampal onset occur regularly and remain stable in frequency and duration for months [39]. These recurrent seizures are mostly confined to the ipsilateral hippocampus with contralateral spread in some animals, but very rare generalizations to the cortex.

In order to assess the feasibility and effect of IntMRT on this model, we first injected kainate into the right hippocampus of C57Bl6 mice, and then placed custom-made bipolar carbon electrodes (MRI compatible) in this structure. Baseline hippocampal recordings were acquired after the four-week epileptogenesis period and focal seizures were quantified. Then, we applied IntMRT to the ipsilateral dorsal hippocampus which was shown to generate seizures [41]. Among the 8 mice irradiated at 100 Gy which displayed seizures before irradiation (Fig. 6A) two presented a decrease of focal seizures at 3 weeks post-irradiation and a complete suppression at 2 and 4 months (Fig. 6C). Two other mice showed a decrease of seizures at 4 months post irradiation. The remaining 4 irradiated epileptic mice did not present any significant decrease of seizures despite histologically identifiable traces of MB (Fig. 6B) and MRI evidence of correct targeting. This lack of efficiency in $50 \%$ of mice must be pondered by the fact that epileptic hippocampus of a MTLE mouse is by definition small, irregularly shaped and located below the cortical surface. The fact that we managed to suppress seizures in $25 \%$ of the animal with no obvious deleterious behavioral effect is however encouraging to try other MRT protocols.

\section{Current and future animal experiments}

From our experience with GAERS rats and MTLE mice, we concluded that IntMRT is a unique tool that allows the deposition of high doses of radiation in restricted volumes with such a precision that a mouse hippocampus can be targeted. However, the consequences of seamless irradiation are not exempt of long-term risks. We therefore decided to pursue our future experiments using the IntMRT method but in such a fashion that only parts of the volume are irradiated. Used as such IntMRT offers the best of two worlds: (i) the deposition of high restricted doses through the MB interlacement, and (ii) the preservation of the vascular system by fractionated irradiations.

Our goal is now to assess the antiepileptic effect of this refined IntMRT method using models of refractory neocortical epilepsies in rats. Indeed, in human this type of epilepsy is frequently resistant to AED, displays considerable comorbidities $[42,43]$ and is surgically 
A

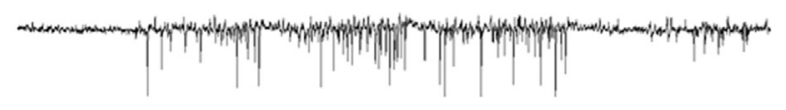

Post-irradiation

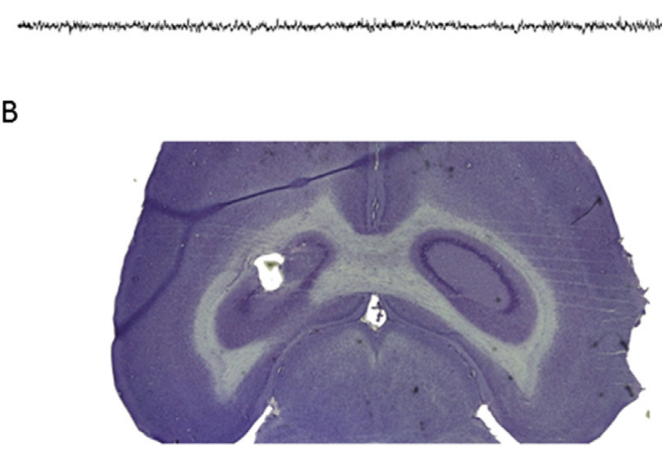

C

Cumulated seizure duration

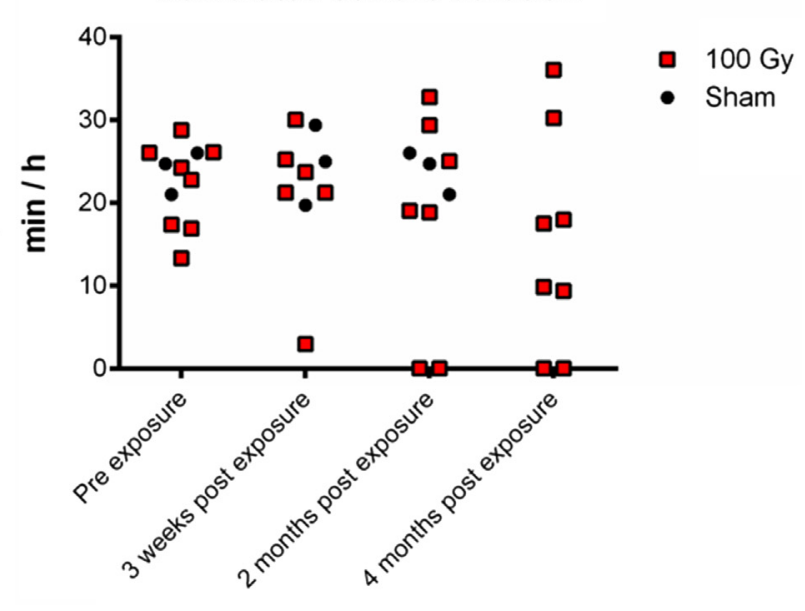

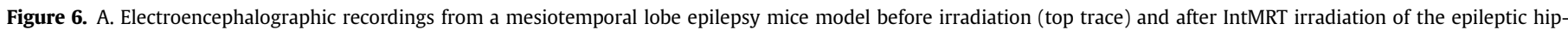

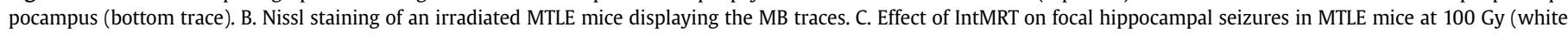
squares, $n=8$ ) and $0 \mathrm{~Gy}$ (black dots, $\mathrm{n}=3$ ). Note that given the electrode technology it was not always possible to record all the mice.

difficult to treat since seizures mostly arise from an eloquent cortical area.

\section{Towards clinical applications}

IntMRT should offer possible clinical applications and our consortium is currently developing pre-clinical studies towards that purpose. However, implementing IntMRT for epileptic patients is not a trivial issue and several aspects need to be addressed when considering the possibilities to develop IntMRT for clinical applications.

\section{Which forms of epilepsy could benefit from IntMRT?}

Resective surgery is currently the gold standard to treat efficiently and even cure patients with refractory epilepsies associated with focal seizures [36]. It has been greatly developed over the last twenty years due to improvements of neuroimaging, electrophysiological and neuropsychological technics (for review see Romanelli et al., 2012 [44]). Yet, such resections are not possible when the epileptogenic zone is close to or belongs to an eloquent cortex area as it would lead to dramatic neurological complications. Similarly, resection of multiple epileptic foci or subcortical ones are difficult and highly risky to perform. These forms of epilepsies are already being treated by stereotactic radiosurgical procedures using Gamma- or Cyber-Knife ${ }^{\circledR}$, in particular when epilepsy is secondary to brain tumors (e.g., arteriovenous malformation, cavernomas; [44]) or hypothalamic hamartomas [45]. Such techniques have also been shown to be efficient for mesiotemporal lobe epilepsy with favorable outcomes [46]. IntMRT will therefore first target these forms of epilepsies for which it would benefit from the experience of radiosurgery. Applying IntMRT to a brain area that will be later removed by surgical resection will very likely be a strategy for a proof-of-concept clinical trial in order to collect anatomical and functional information on the resected tissue to better understand the consequences of irradiation, while reducing the risks for the patients. Given our first data in animal models, it is likely that IntMRT will improve radiosurgical treatment of refractory epilepsies and/or reduce their side-effects. In addition to epilepsies associated with focal seizures, IntMRT should also allow to consider drug-resistant or difficult to treat generalized epilepsies such as the
Landau-Kleffner syndrome, a severe form of child epilepsy which is associated with aphasia and continuous spike-and-wave during sleep [47]. For this particular syndrome, multiple subpial transections have been proposed [48] which aim at disconnecting horizontal interneurons to reduce the synchronization of principal ones. Although good outcomes have been obtained [49] using this technic, it is greatly invasive and represents an important risk for the patients. IntMRT, by allowing very precise deposition of high doses should allow to reproduce subpial transection and/or the specific alteration of horizontal interneurons. Proof-of-concept experiments in animal models should allow us to determine whether IntMRT could be used for such approach.

\section{The need for other mammalian models}

Although quite challenging, it is necessary to develop methods which allow the irradiation of larger brains such as those of human patients. In the case of epilepsy, the application of high-flux fractionated $\mathrm{MB}$ in mammalian models other than rodents appears necessary before considering its application to humans. Indeed, such trials should provide evidence for antiepileptic effects of MB in species with larger brain volume and more complex symptoms and comorbidities associated with the epilepsy. One major issue for a clinical application is to take into account the complex shape of the human cortex, i.e. the gyri and sulci forming the cortical convolutions. This is indeed a real challenge as the angle of irradiation may vary according to the localization of the cortical focus to target. To this aim the use of non-human primates appears the most appropriate as their cortical architecture and somatotopic arrangement is closer to human patients [50]. Furthermore, predictive models of epilepsy have been described in macaques which should allow preclinical evaluation of the efficacy of MB [51,52]. In addition, such models would be more efficient to address the behavioral side effects of synchrotron MB irradiation, since sophisticated behavioral and cognitive tests exist in non-human primates which are much closer to human competencies than in other mammalian species. For instance, when MB irradiation targets a brain region dedicated to the motor control of the hand, behavioral tests exists in nonhuman primate to quantify the hand dexterity [53], a critical control that cannot be addressed in other species. The use of nonhuman primates should, however, be limited as much as possible 
and one should consider only models with a validated clinical significance [54]. This can be envisioned at ESRF because of the existing irradiation infrastructures including an advanced patient safety system, the availability of suitable x-ray microbeams, the presence of skilled personnel and the availability of a biomedical facility near the irradiation room. Indeed, to develop new irradiation therapies it is critical to combine a clinical expertise in the surgery and radiosurgery of epilepsy.

\section{Dosimetric and safety issues}

The non-human primate studies are also essential to address the questions of dosimetric and safety issues in the purpose of human patients treatment. Studies using magnetic resonance imaging show periodic displacements in the brain of up to $1 \mathrm{~mm}$ in humans [55]. In the case of irradiation of non-human primates, the cyclic motion of the brain, induced by cardiac vascular movements and respiratory pulsations of the cerebrospinal fluid, imposes efforts in addition to those taken on the treatment planning for interlaced microbeams in rodent animal models [56]. Since only one port can be irradiated at a time, these movements must be understood in detail to make them predictable and ensure that irradiations from all ports happen at exactly the same point of the cyclic motion. Otherwise the precisely interlaced beams may be shifted with reference to each other and create gaps or overlapping areas leading to an inhomogeneous dose deposition in the target area. Monte Carlo dose calculations shall be used to accurately plan doses applied to non-human primates, including effects of dose blurring due to brain movement within one irradiation and movements in between different ports. The results will need to be cross-checked with experimental results from the irradiation of a suitable phantom able to reproduce the fine movements of a human brain. Using non-human primates appears therefore necessary to implement and validate all instrumental, dosimetric, safety and ethical aspects to become as close as possible to clinical standards.

\section{Hospital implementation}

The European synchrotron X-ray source in Grenoble is for now the only place in the World where preclinical trials using IntMRT are carried out on a regular time basis. The first technical developments of high flux synchrotron X-ray microbeams have been carried out for preclinical brain tumor radiation therapy. This research domain is still active and promising enough for a realistic clinical transfer in the years to come. The later application to the epilepsy presented here reinforces the interest of such X-ray sources in radiation therapy domain. These trials requires state-ofthe-art technical developments for imaging and X-ray dosimetry control and are foreseen in others national or international X-ray synchrotron sources. The encouraging preclinical results shown here pave the way for future clinical applications using machines available in clinics, i.e. outside the synchrotron scientific community. The potential use of microbeam X-ray radiosurgery in the hospital environment will depend mainly on the ability of new table-top X-ray sources to produce sufficient dose-rates to perform radiosurgical procedures at acceptable cost.

Different projects aiming to build such table-top powerful X-ray sources have been funded at least in three countries: US, Germany and France. As a result extensive preclinical trials using this tabletop X-ray sources are to be encouraged and foreseen.

\section{Conclusions}

In this article we review several studies in animal models of epilepsy which show the great interest of Interlaced Microbeam
Radiotherapy to block the synchronization of epileptic neuronal networks. These studies showed also the limits of these techniques by revealing the variability of their application to small irregularly shaped sub-cortical structures in the mice which do not allow a sufficient targeting accuracy. There is now a need to continue to apply IntMRT to rat models of epilepsy closer to the more severe forms of human epilepsy and to implement such radiosurgery approach to non-human primate models of epilepsy as a proof-ofconcept of its feasibility in human patients.

\section{Acknowlegments}

This work was supported by Institut National de la Santé et de la Recherche Médicale (INSERM), a grant from Agence Nationale de la Recherche (ANR "EPIRAD" n ${ }^{\circ}$ ANR-13-BSV1-0012-01), European Synchrotron Research Facility (ESRF) and the COST Action TD1205 (SYRA3).

\section{References}

[1] Olesen J, Gustavsson A, Svensson M, Wittchen HU, Jönsson B, on behalf of the CDBE2010 Study Group. The economic cost of brain disorders in Europe. Eur J Neurol 2011;19:155-62.

[2] Kwan P, Brodie MJ. Early identification of refractory epilepsy. N Engl J Med 2000;342:314-9.

[3] ANAES. Prise en charge des épilepsies partielles pharmaco-résistantes. Haute Autorité de Santé; 2004. p. 1-27.

[4] Kahane P. La chirurgie de l'épilepsie chez l'adulte: pour qui? Rev Neurol 2004;160:179-84. Spec No 1.

[5] Régis J, Rey M, Bartolomei F, Vladyka V, Liscak R, Schröttner O, et al. Gamma knife surgery in mesial temporal lobe epilepsy: a prospective multicenter study. Epilepsia 2004;45:504-15.

[6] Romanelli P, Anschel DJ. Radiosurgery for epilepsy. Lancet Neurol 2006;5: 613-20.

[7] Sims E, Doughty D, Macaulay E, Royle N, Wraith C, Darlison R, et al. Stereotactically delivered cranial radiation therapy: a ten-year experience of linacbased radiosurgery in the UK. Clin Oncol R Coll Radiol 1999;11:303-20.

[8] St George EJ, Kudhail J, Perks J, Plowman PN. Acute symptoms after gamma knife radiosurgery. J Neurosurg 2002;97:631-4.

[9] Romanelli P, Bravin A. Synchrotron-generated microbeam radiosurgery: a novel experimental approach to modulate brain function. Neurol Res 2011;33(8):825-31.

[10] Zeman W, Curtis HJ, Gebhard KL, Haymaker W. Tolerance of mouse brain tissue to high energy deuterons. Science 1959;130:1760-1.

[11] Curtis HJ. The interpretation of microbeam experiments for manned space flight. Radiat Res Suppl 1967;7:258-64.

[12] Curtis HJ. The use of deuteron microbeam for simulating the biological effects of heavy cosmic-ray particles. Radiat Res Suppl 1967:7:250-7.

[13] Zeman W, Curtis HJ, Baker CP. Histopathologic effect of high-energy-particle microbeams on the visual cortex of the mouse brain. Radiat Res 1961;15: 496-514.

[14] Anschel DJ, Bravin A, Romanelli P. Microbeam radiosurgery using synchrotron-generated submillimetric beams: a new tool for the treatment of brain disorders. Neurosurg Rev 2011;34:133-42.

[15] Slatkin D, Spanne P, Dilmanian F, Sandborg M. Microbeam radiation-therapy. Med Phys 1992;19:1395-400.

[16] Slatkin DN, Spanne P, Dilmanian FA, Gebbers JO, Laissue JA. Subacute neuropathological effects of microplanar beams of x-rays from a synchrotron wiggler. Proc Natl Acad Sci U S A 1995;92:8783-7.

[17] Laissue JA, Blattmann H, Di Michiel M, Slatkin DN, Lyubimova N, Guzman R, et al. The weanling piglet cerebellum: a surrogate for tolerance to MRT (microbeam radiation therapy) in pediatric neuro-oncology. In: Proc. SPIE 4508, Penetrating Radiation Systems and Applications III; 2001. p. 65.

[18] Serduc R, Bräuer-Krisch E, Siegbahn EA, Bouchet A, Pouyatos B, Carron R, et al. High-precision radiosurgical dose delivery by interlaced microbeam arrays of highflux low-energy synchrotron X-rays. PLoS One 2010;5:e9028.

[19] Prayson RA, Yoder BJ. Clinicopathologic findings in mesial temporal sclerosis treated with gamma knife radiotherapy. Ann Diagn Pathol 2007;11:22-6.

[20] Rheims S, Didelot A, Guenot M, Régis J, Ryvlin P. Subcontinuous epileptiform activity after failed hippocampal radiosurgery. Epilepsia 2011;52:1425-9.

[21] Mori Y, Kondziolka D, Balzer J, Fellows W, Flickinger JC, Lunsford LD, et al. Effects of stereotactic radiosurgery on an animal model of hippocampal epilepsy. Neurosurgery 2000;46:157-65. discussion 165-8.

[22] Maesawa S, Kondziolka D, Dixon CE, Balzer J, Fellows W, Lunsford LD. Subnecrotic stereotactic radiosurgery controlling epilepsy produced by kainic acid injection in rats. J Neurosurg 2000;93:1033-40.

[23] Chen Z, Kamiryo T, Henson S, Yamamoto H, Bertram E, Schottler F, et al, Anticonvulsant effects of gamma surgery in a model of chronic spontaneous limbic epilepsy in rats. J Neurosurg 2001;94:270-80. 
[24] Quigg M, Rolston J, Barbaro NM. Radiosurgery for epilepsy: clinical experience and potential antiepileptic mechanisms. Epilepsia 2012;53:7-15.

[25] Pouyatos B, Serduc R, Chipaux M, Chabrol T, Bräuer-Krisch E, Nemoz C, et al. Synchrotron X-ray interlaced microbeams suppress paroxysmal oscillations in neuronal networks initiating generalized epilepsy. Neurobiol Dis 2013;51: 152-60.

[26] Danober L, Deransart C, Depaulis A, Vergnes M, Marescaux C. Pathophysiological mechanisms of genetic absence epilepsy in the rat. Prog Neurobiol 1998:55:27-57.

[27] Depaulis A, Van Luijtelaar EL. Genetic models of absence epilepsy in the rat. In: Pitkänen A, Schwartzkroin PA, Moshé SL, editors. Models of seizures and epilepsy. London: Elsevier; 2005. p. 233-48.

[28] Polack PO, Guillemain I, Hu E, Deransart C, Depaulis A, Charpier S. Deep layer somatosensory cortical neurons initiate spike-and-wave discharges in a genetic model of absence seizures. J Neurosci 2007;27:6590-9.

[29] David O, Guillemain I, Saillet S, Reyt S, Deransart C, Segebarth C, et al. Identifying neural drivers with functional MRI: an electrophysiological validation. PLoS Biol 2008;6:2683-97.

[30] Paxinos G, Watson C. The rat brain in stereotaxic coordinates. 2007. p. 456.

[31] Siegbahn EA, Bräuer-Krisch E, Bravin A, Nettelbeck H, Lerch ML, Rosenfeld AB, et al. MOSFET dosimetry with high spatial resolution in intense synchrotron generated X-ray microbeams. Med Phys 2009;36:1128-37.

[32] Serduc R, Vérant P, Vial JC, Farion R, Rocas L, Rémy C, et al. In vivo twophoton microscopy study of short term effects of microbeam irradiation on normal mouse brain microvasculature. Int J Radiat Oncol Biol Phys 2006;64: $1519-27$.

[33] Yoshii Y, Phillips TL. Late vascular effects of whole brain X-irradiation in the mouse. Acta Neurochir 1982;64:87-102.

[34] Münter MW, Karger CP, Reith W, Schneider HM, Peschke P, Debus J. Delayed vascular injury after single high-dose irradiation in the rat brain: histologic, immunohistochemical and angiographic studies. Radiology 1999;212: 475-82.

[35] Hamelin S, Depaulis A. Revisiting hippocampal sclerosis in mesial temporal lobe epilepsy according to the "two-hit hypothesis". Rev Neurol 2015;171: 227-35.

[36] Wiebe S, Blume WT, Girvin JP, Eliasziw M, Effectiveness and Efficiency of Surgery for Temporal Lobe Epilepsy Study Group. A randomized, controlled trial of surgery for temporal-lobe epilepsy. N Engl J Med 2001;345:311-8.

[37] Depaulis A, Hamelin S. Animal models for mesiotemporal lobe epilepsy: the end of a misunderstanding? Rev Neurol 2015;171:217-26.

[38] Suzuki F, Junier MP, Guilhem D, Sørensen JC, Onteniente B. Morphogenetic effect of kainate on adult hippocampal neurons associated with a prolonged expression of brain-derived neurotrophic factor. Neuroscience 1995;64: 665-74.

[39] Riban V, Bouilleret V, Phâm-Lê BT, Fritschy JM, Marescaux C, Depaulis A. Evolution of hippocampal epileptic activity during the development of hippocampal sclerosis in a mouse model of temporal lobe epilepsy. Neuroscience 2002;112:101-11.
[40] Heinrich C, Nitta N, Flubacher A, Müller M, Fahrner A, Kirsch M, et al. Reelin deficiency and displacement of mature neurons, but not neurogenesis, underlie the formation of granule cell dispersion in the epileptic hippocampus. J Neurosci 2006;26:4701-13.

411] Pallud J, Häussler U, Langlois M, Hamelin S, Devaux B, Deransart C, et al. Dentate gyrus and hilus transection blocks seizure propagation and granule cell dispersion in a mouse model for mesial temporal lobe epilepsy. Hippocampus 2011;21:334-43.

[42] Sander JW. The epidemiology of epilepsy revisited. Curr Opin Neurol 2003;16: $165-70$.

[43] Thomas JE, Reagan TJ, Klass DW. Epilepsia partialis continua. A review of 32 cases. Arch Neurol 1977;34:266-75.

[44] Romanelli P, Striano P, Barbarisi M, Coppola G, Anschel DJ. Non-resective surgery and radiosurgery for treatment of drug-resistant epilepsy. Epilepsy Res 2012;99:193-201.

[45] Régis J, Scavarda D, Tamura M, Nagayi M, Villeneuve N, Bartolomei F, et al. Epilepsy related to hypothalamic hamartomas : surgical management with special reference to gamma knife surgery. Childs Nerv Syst 2006;22:881-95.

[46] Bartolomei F, Hayashi M, Tamura M, Rey M, Fischer C, Chauvel P, et al. Longterm efficacy of gamma knife radiosurgery in mesial temporal lobe epilepsy. Neurology 2008;70(19):1658-63.

[47] Rotenberg J, Pearl PL. Landau-Kleffner syndrome. Arch Neurol 2003;60(7): 1019-21.

[48] Morrell F, Whisler WW, Bleck TP. Multiple subpial transection: a new approach to the surgical treatment of focal epilepsy. J Neurosurg 1989;70:231-9.

[49] Spencer SS, Schramm J, Wyler A, O'Connor M, Orbach D, Krauss G, et al Multiple subpial transection for intractable partial epilepsy: an international meta-analysis. Epilepsia 2002;43:141-5

[50] Sessle BJ, Wiesendanger M. Structural and functional definition of the motor cortex in the monkey (Macaca fascicularis). J Physiol 1982;323:245-65.

[51] Ribak CE, Seress L, Weber P, Epstein CM, Henry TR, Bakay RA. Alumina gel injections into temporal lobe of rhesus monkeys cause complex partial seizures and morphological changes found in human temporal lobe epilepsy. J Comp Neurol 1998;401(2):266-90.

[52] Devergnas A, Piallat B, Prabhu S, Torres N, Benabid AL, David O, et al. The subcortical hidden side of focal motor seizures: evidence from microrecordings and local field potentials. Brain 2012;135:2263-76.

[53] Schmidlin E, Kaeser M, Gindrat AD, Savidan J, Chatagny P, Badoud S, et al. Behavioral assessment of manual dexterity in non-humanprimates. J Vis Exp 2011;57:3258.

[54] Guillemain I, Kahane P, Depaulis A. Animal models to study aetiopathology of epilepsy: what are the features to model? Epileptic Disord 2012;14:217-25.

[55] Maier SE, Hardy CJ, Jolesz FA. Brain and cerebrospinal fluid motion: real-tim quantification with M-mode MR imaging. Radiology 1994;193(2):477-83.

[56] Kao YH, Guo WY, Liou AJK, Chen TY, Huang CC, Chou CC, et al. Transfer function analysis of respiratory and cardiac pulsations in human brain observed on dynamic magnetic resonance images. Comput Math Methods Med 2013:157040. 